\title{
Constructing Patient Specific Models for Correcting Intraoperative Brain Deformation
}

\author{
A.D. Castellano-Smith ${ }^{1}$, T. Hartkens ${ }^{1}$, J. Schnabel ${ }^{1}$, D.R. Hose ${ }^{2}$, H. Liu ${ }^{3}$, W.A. Hall ${ }^{3}$, \\ C.L. Truwit ${ }^{3}$, D.J. Hawkes ${ }^{1}$, and D.L.G. Hill ${ }^{1}$ \\ ${ }^{1}$ Computational Imaging Sciences Group, Radiological Sciences, Guy's Hospital, King's \\ College London, London SE1 9RT, UK \\ Andrew.Castellano-Smith@kcl.ac.uk \\ 2 Medical Physics Department, Clinical Sciences Division, University of Sheffield, UK. \\ 3 Depts. of Radiology and Neurosurgery, University of Minnesota, Minneapolis, MN, USA.
}

\begin{abstract}
In this work we present a Mesh Warping technique for the construction of patient-specific Finite Element Method models from patient MRI images, and demonstrate how simulated surgical loading can be applied to these models. We compare the results of this simulation with observed deformation during surgery, and show that our model matches well with the observed degree of deformation.
\end{abstract}

\section{Introduction}

Brain deformation during neurosurgery can substantially degrade the utility of pre-operative imaging as a surgical guidance tool. Structures on or below the surface of the brain have been found to move by $10 \mathrm{~mm}$ or more [1].

Biomechanical models have the potential to predict brain deformation and may in future, be incorporated into image guided surgery systems to improve accuracy [2]. For these models to be successful, they are likely to need to treat different parts of the brain in different ways. The generation of patient-specific brain models is extremely time-consuming, as it requires segmentation of relevant structures within the patient, generation of a 3D mesh with appropriate properties allocated to each element, and finally solving the model using suitable conditions.

We propose a technique for building a finite element model of an individual patient by non-rigid registration of a carefully meshed atlas image to the pre-operative image of a specific subject. We demonstrate this meshing approach on 4 patients. Once the data mesh has been generated, each element in the mesh can be assigned appropriate mechanical properties (including any expected volume change), and then the finite element model is solved after applying appropriate forces.

\section{Methods}

\subsection{Patient Selection and Imaging}

To assess the effectiveness of the techniques used in this paper, we selected patients who were undergoing surgery at the interventional MR facility at the University of 
Minnesota. Typical scanning protocol consisted of acquiring multi-slice spin echo and MP-RAGE volume datasets prior to commencement of surgery, similar acquisitions immediately after surgery was completed while the patient is still in the operating position. Additional single-slice images were acquired intra-operatively.

From this set of patients (13 resection, 5 biopsy, 4 functional procedures), we selected four patients ( 2 resection cases, 2 functional surgery cases) to demonstrate our mesh warping algorithm. We then simulate deformation on one of these subjects (a functional surgery patient), comparing the modelled deformation with the deformation imaged at the end of surgery.

\subsection{Quantification of CSF Volume Change During Surgery}

For the 21 patients listed above, we estimated CSF volume loss during the procedure by manually segmenting the lateral ventricles from the pre- and post-surgery images.

\subsection{FEM Model Generation - Mesh Warping}

The use of Finite Element Methods (FEMs) for modelling brain deformation during surgery has been the subject of some study [2]3,4]. In order to simulate surgical loads on a patient dataset, it is necessary to construct a patient-specific FEM model incorporating salient features of that particular patient geometry. However, the construction of FEM meshes fitting to the features in an MR image of a head is very difficult and time-consuming. To model each patient head and brain in a realistic fashion, we have devised a technique for constructing FEM meshes based on an atlas mesh and a nonrigid registration technique, which we refer to as Mesh-Warping.

In this technique, the construction of patient-specific FEM meshes relies on the construction of a mesh fitting to an atlas brain. This mesh is then deformed according to the non-rigid registration solution found by registering the patient image to the atlas brain image. This technique is similar to the Mesh-Matching technique discussed in [5] but in our case, the whole image is used during the registration procedure as opposed to the surface-based registration in [5], providing a better fitting patient mesh.

Atlas Model The Brainweb brain atlas 1 was used as our brain model. In order to construct a FEM model from the segmented images in the atlas, surface models were constructed of the outer brain surface (grey-matter/CSF boundary) and the ventricular surfaces. These surface models were extracted from the images using a modified marching cubes technique [6] before being smoothed and decimated to remove small edges and small triangular facets using the "evolver" software package 2 . This smoothing and decimation process has been shown [7] to preserve salient features of surface shape, whilst providing a reduction in the number of facets making up the model surface.

The resulting surface models (brain surface and ventricles) were then loaded into ANSY $\$ 3$, a commercial FEM package we have used in previous work, the interior volumes of the surfaces constructed, and a combined volume produced. The techniques

\footnotetext{
${ }^{1}$ from http://www.bic.mni.mcgill.ca/brainweb/

${ }^{2}$ from http://www.susqu.edu/facstaff/b/brakke/evolver/evolver.html

${ }^{3}$ ANSYS Inc. http://www.ansys.com
} 
presented in this paper do not rely on the use of a specific FEM package. This multicomponent volume was then meshed with tetrahedral elements. The effect of meshing in this way is to preserve the internal boundaries within the object - in this case the ventricular surfaces, whilst producing a continuous mesh across that surface. The smoothed surface of the grey matter and ventricles is preserved within the mesh.

Segmenting white matter separately and constructing a surface model as for the grey matter and ventricular surfaces was not practicable, because the brainweb white matter is non-connected. In order to allow for the different biomechanical properties of grey matter, white matter and CSF we therefore chose to label the mesh with tissue-type labels after meshing. This labelling assigns to each tetrahedral element in the mesh a tissue type - grey matter, white matter or fluid. The result of such a labelling can be seen in Figure 1.

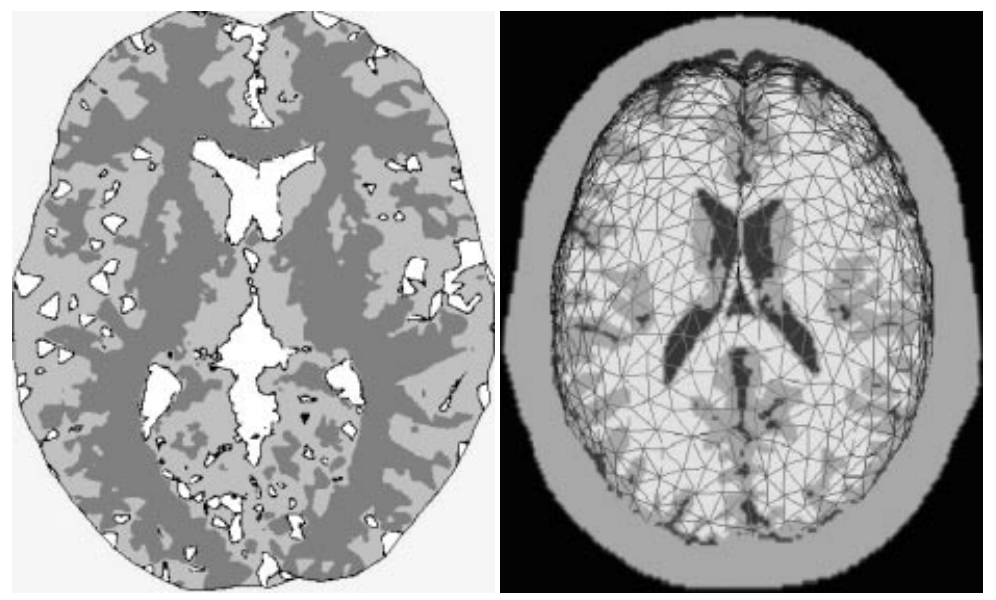

Fig. 1. The atlas mesh, labelled (Left) with fluid (white), white matter (dark grey) and grey matter (light grey). The ventricular surface was segmented and constructed separately, and is preserved in the mesh, whereas white matter regions are labelled within the mesh after construction. The atlas mesh (right) is displayed as a wireframe overlaid on an atlas slice.

Non-rigid Registration for Mesh Warping In order to construct a subject-specific mesh for each patient in the study, it would of course be possible to follow the process described for the construction of the atlas mesh, but for each patient. However, this process is slow and labour intensive - manual segmentation of ventricular surfaces, grey and white matter is required. Decimation and smoothing of the surface models requires user interaction, and meshing is computationally expensive, requiring several hours. The construction of the atlas mesh used in this work required several tens of hours from start to finish. Clearly this time-scale is clinically unacceptable, and for this reason, we propose a method for patient-specific mesh construction which avoids these problems. 
The fully automated non-rigid registration in use in our centre [8.9] can accurately align the ventricular surfaces, the cortical surface and other salient features. This registration procedure produces a deformation field relating all points in the target image (in this case the atlas) to a position in the source image (the patient). In this way, the atlas mesh can be warped to fit the patient geometry. We believe that the use of a nonrigid intensity-based registration scheme to warp meshes to fit patient data has not been used before and has great potential for allowing patient-specific FEM models to be constructed in a clinically relevant timescale.

The warped, patient-specific mesh is only suitable for use if the elements produced meet quality criteria. Any folding during the registration procedure can produce elements which are also folded, and hence do not usefully model the patient geometry. Other "badly shaped" elements, such as tetrahedra with very extreme angles, must also be avoided. ANSYS provides mesh checking capabilities to identify such elements, and manual correction of the mesh can then be made. This correction process could be automated, if required, based on an examination of the mesh element surface normals.

Each of the four patients in the study was registered to the atlas image, and the prelabelled atlas mesh then warped following the deformation field produced. The mesh quality was checked, in each case, for folding and badly shaped elements. These were manually corrected before further processing.

\subsection{Simulation Scenarios}

To simulate the surgical situation, we firstly fixed the position of all external points on the grey matter surface, simulating the connection of the brain to the inside of the skull by the arachnoid strands. The patient selected for modelling had a functional procedure involving no resection.

The Young's Modulus of the tissues, following [10] were set to $4 \times 10^{3} \mathrm{NM}^{-2}$ for white matter, and $8 \times 10^{3} \mathrm{NM}^{-2}$ for grey matter, with Poisson's ratio of 0.495 for near incompressibility. All tissues were modelled as linear elastic solids using 10-noded tetrahedral elements (the "solid187" element in ANSYS). The measurements of ventricular volume suggested that we should simulate CSF loss by shrinking the lateral ventricles by $10 \%$ during the procedure. The region of brain surface apendent to the electrode entry point was allowed to move freely on the side ipsi-lateral to the insertion. We then applied gravity to the model (a uniform vertical force on each element) and the ventricles were reduced in volume by $10 \%$ by applying a thermal load to the FEM mesh elements making up the ventricular system such that they uniformly shrink in volume by $10 \%$.

Comparison of Real and Simulated Deformations The FEM solution was used to produce a warped version of the pre-operative image, simulating an MR image of the post-operative brain. We visually compared this simulated image with the real intraoperatively recorded image data. 


\section{Results}

\subsection{Quantification of Deformation}

Volume Vhange of Ventricles The ventricle ipsi-lateral to the craniotomy exhibites larger volume changes than the contra-lateral ventricle in cases where a substantial difference in volume change occurs between the ventricles. Larger volume changes occur in the resection cases than in the biopsy or functional cases. The variability in volume change measure assessed by repeated segmentation of a pre/post-operative ventricle image pair was $5.4 \%$. This variability is similar to the variability in segmentation of cerebral hemispheres in earlier work [7].

Finite Element Meshes The patient-specific meshes for the four patients in this study are shown in Figure 2 Near some gyri the mesh is not aligned perfectly with the cortical surface, due to shrinkage of the mesh surface during the decimation used in the atlas construction. This should not cause large errors in the solutions, and will be addressed by the construction of a finer model.
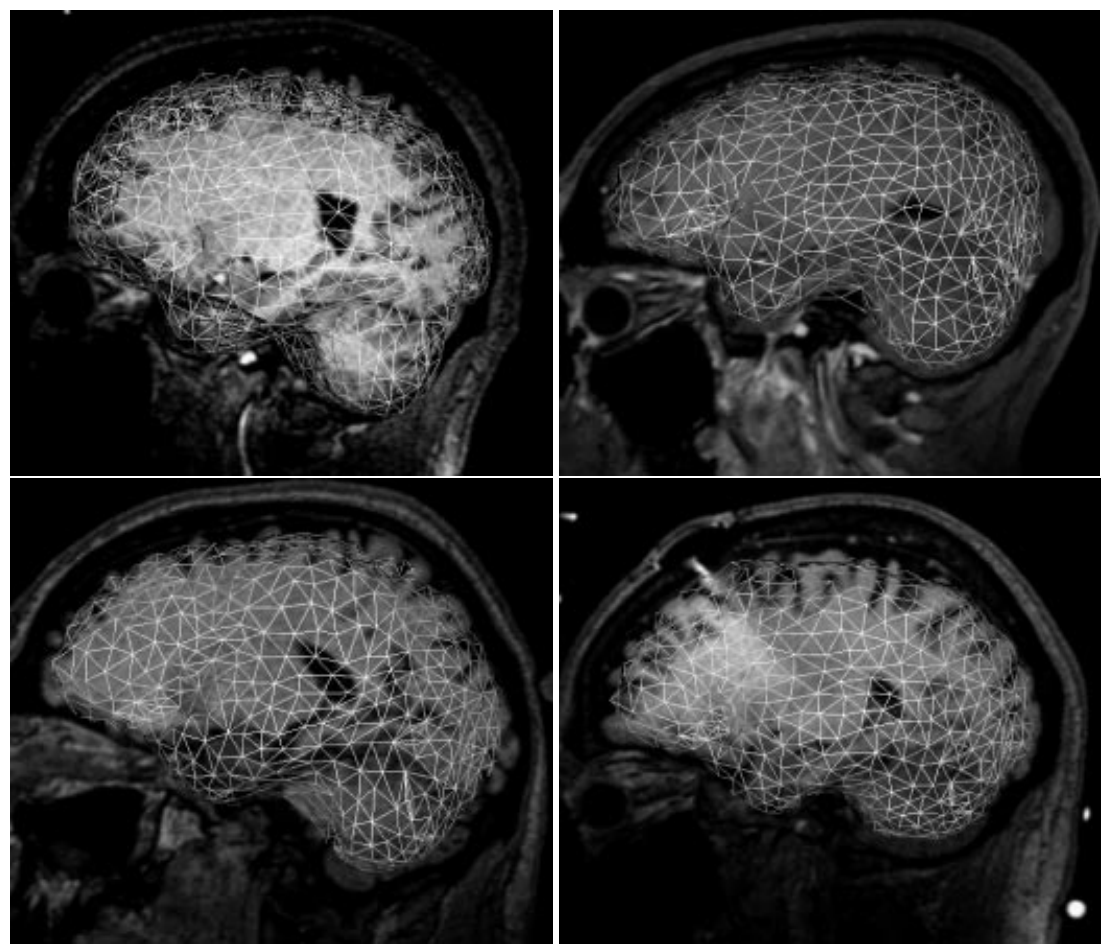

Fig. 2. FEM meshes of the four patients in the study. Top left - functional2; Top right resection7; Bottom left - resection11; Bottom right - functional6. There is some shrinkage of the mesh away from the cortical surface due to the decimation and smoothing applied to the atlas model. 
The meshes each contain 56747 nodes and 40310 tetrahedral elements - the same number as the atlas mesh. The mesh quality can be quantified by the number of folded and badly shaped elements in the mesh due to the registration and Mesh Warping procedure. In all cases, fewer than 20 elements were badly shaped. Only resection7 had a substantial number of folded elements (6276), caused by the abnormal structure of the brain making registration to the atlas difficult.

\subsection{Comparison of FEM Solutions with Quantification of Brain Deformation}

In Figure 3 the top two images show the FEM model after deformation, with the displacement of brain surface colour coded, lightest colour corresponds to $4.5 \mathrm{~mm}$ displacement. The middle image pair shows the subtraction of the post-surgery image from the pre-operative image. The lower images show subtraction of the simulated post-surgery image from the pre-operative image. Note that the deformation of the frontal lobes is greater ipsi-lateral to the electrode insertion in both cases, and that the ventricular volume change appears visually similar in both the real and simulated images. The simulated data lacks the noise and RF inhomogeneity artefacts of the real data.
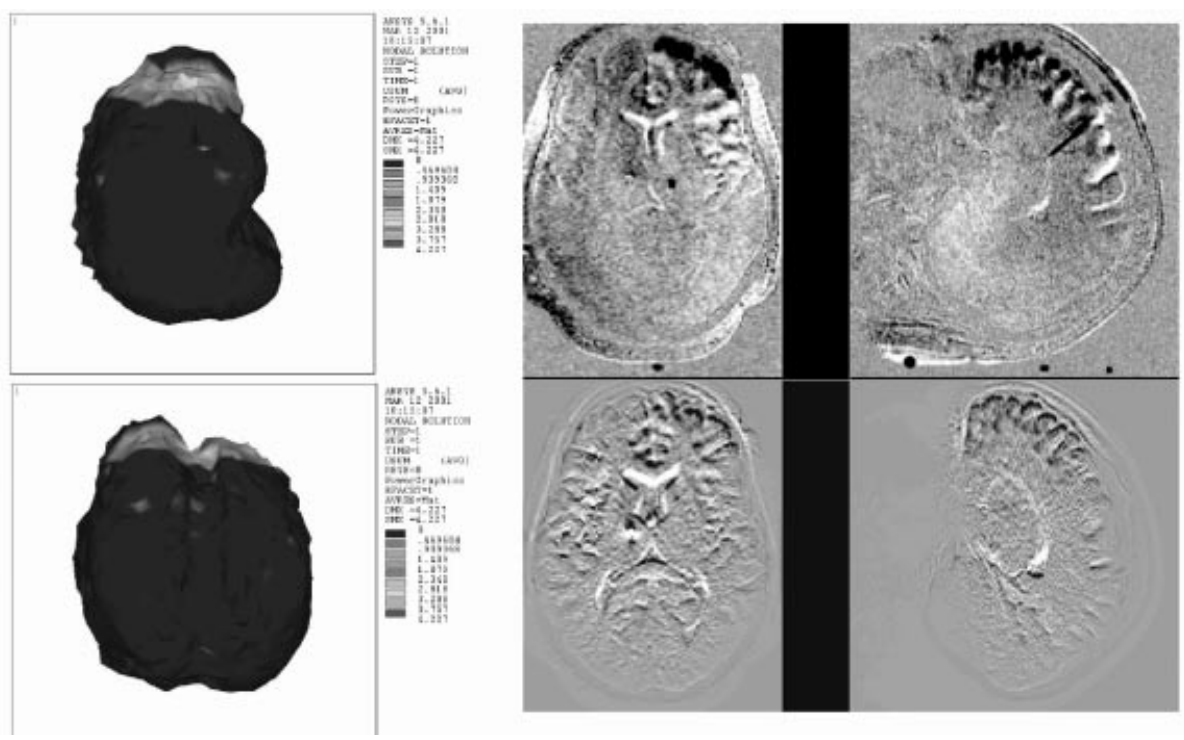

Fig. 3. Comparison of modelled deformation to true deformation. Top panels show the deformed FEM model (colour animated version of this at http://wwwipg.umds.ac.uk/a.d.smith/miccai2001/). Middle images show subtraction of postsurgery image from pre-operative image. Bottom images show subtraction of simulated post-operative image from pre-operative image. 


\section{Conclusions and Discussion}

In this paper we have described a technique to generate a patient-specific finite element model for predicting intraoperative brain deformation. This technique, which we refer to as Mesh Warping, involves 3D registration of an atlas image that has been carefully meshed to a pre-operative MR image of the subject being studied. This assigns properties to grey matter, white matter and CSF in the model. We have applied this meshing technique to four neurosurgical subjects imaged in the interventional MR facility at the University of Minnesota. For one of these patients who had a functional surgical procedure, we then ran an example deformation scenario in which CSF volume was reduced by $10 \%$, the attachment of the brain to the dura was broken appendant to the point of insertion of the electrode. We show that the model generates visually plausible deformation. The run time of the algorithm was 4 hours on a $300 \mathrm{MHz}$ Sun Ultra 10 using the commercial ANSYS finite element modelling software. Running the algorithm on a state-of-the art CPU may in the near future give a run-time that is compatible with the timescale of neurosurgical procedures.

Meshing with no user interaction saves a lot of time. The registration may in some cases be slow, but is fully automated. Our Mesh-Warping technique is not limited to the non-rigid registration technique in use in our centre, but may be applied using any suitable non-rigid registration algorithm.

Further work will involve the construction of a higher resolution atlas model, incorporating more detail of brain folds. The fidelity of the model's deformation predictions will be assessed in future work involving more intra-operative data collection during neurosurgical procedures to provide better localization of deformation. This work will also allow the testing of these techniques on a much larger set of patients. The interaction between sulcal walls will become important in this model, as deformations within the FEM model will need to take account of the unrealistic nature of deformations involving tissue moving through other tissue. All the FEM solutions presented here are equilibrium state solutions. We will explore the possibility of finding temporally varying solutions to allow a time-varying deformation to be predicted. More use of the intraoperative image data will allow a verification of the predictions made by this model.

We assume that the brain surface gravitationally lower than or contralateral to the craniotomy remains firmly tethered to the inner table of the skull. The degree to which this assumption is true in practice needs investigation, but displacements of these areas of brain surface, observationally at least, appear small compared to the displacements of other structures.

The simulation of actual resections by removing sections of brain from the mesh, remains a challenge to the current model which we are addressing in on-going work.

The measurement of mechanical properties of biological tissues is difficult, and more especially where they relate to abnormal tissues. In this work we have used literature values. Future work will assess the sensitivity of the model's solutions to differences in material properties over the ranges found in the literature. 


\section{Acknowledgements}

We are grateful to the UK Engineering and Physical Sciences Research Council for funding ADCS and TH, Justin Penrose of Sheffield University for assistance with ANSYS and colleagues in CISG.

\section{References}

1. D. L. G. Hill, C. R. Maurer, Jr., R. J. Maciunas, J. A. Barwise, J. M. Fitzpatrick, and M. Y. Wang. Measurement of intraoperative brain surface deformation under a craniotomy. Neurosurgery, 43:514-528, 1998.

2. M. I. Miga, K. D. Paulsen, J. M. Lemery, S. D. Eisner, A. Hartov, F. E. Kennedy, and D. W. Roberts. Model-updated image guidance: initial clinical experiences with gravity-induced brain deformation. IEEE Trans. Med. Imaging, 18:866-874, 1999.

3. M. Bro-Neilsen. Finite element modelling in surgery simulation. Proceedings of the IEEE, 86:490-503, 1998.

4. M. I. Miga, K. D. Paulsen, P. J. Hoopes, F. E. Kennedy, A. Hartov, and D. W. Roberts. In vivo quantification of a homogeneous brain deformation model for updating preoperative images during surgery. IEEE Trans. Biomed. Eng., 47:266-273, 2000.

5. B. Couteau, Y. Payan, and S. Lavallee. The mesh-matching algorithm: an automatic $3 \mathrm{~d}$ mesh generator for finite element structures. Journal of Biomechanics, 33:1005-1009, 2000.

6. W.E Lorensen and H.E. Cline. Marching cubes: a high resolution $3 \mathrm{~d}$ surface construction algorithm. Computer Graphics, 21(4):163-169, 1987.

7. A.D. Castellano Smith. The Folding of the Human Brain: From Shape to Function. PhD thesis, King's College London, September 1999.

8. D. Rueckert, L.I. Sonoda, C. Hayes, D.L.G. Hill, M.O. Leach, and D.J. Hawkes. Non-rigid registration using free-form deformations: Application to breast MR images. IEEE Trans. Med. Imaging, 18:712-721, 1999.

9. J. A. Schnabel, D. Rueckert, M. Quist, J. M. Blackall, A. D. Castellano Smith, T. Hartkens, G. P. Penney, W. A. Hall, H. Liu, C. L. Truwit, F. A. Gerritsen, D. L. G. Hill, and D. J. Hawkes. A generic framework for non-rigid registration based on non-uniform multi-level free-form deformations. Proc. MICCAI 2001, Springer LNCS:(in press), 2001.

10. H. Takizawa, K. Sugiura, M. baba, and J. D. Miller. Analysis of intracerebral hematoma shapes by numerical computer simulation using the finite element method. Neurol Med Chir (Tokyo), 34:65-69, 1994. 